\title{
MODEL-BASED COST-EFFECTIVENESS OF CONVENTIONAL AND INNOVATIVE CHEMO- RADIATION IN LUNG CANCER
}

Citation for published version (APA):

Bongers, M. L., de Ruysscher, D., Oberije, C., Lambin, P., Uyl-de Groot, C. A., Belderbos, J., \& Coupe, V. M. H. (2017). MODEL-BASED COST-EFFECTIVENESS OF CONVENTIONAL AND INNOVATIVE CHEMO-RADIATION IN LUNG CANCER. International Journal of Technology Assessment in Health Care, 33(6), 681-690. https://doi.org/10.1017/S0266462317000939

Document status and date:

Published: 01/01/2017

DOI:

10.1017/S0266462317000939

Document Version:

Publisher's PDF, also known as Version of record

Document license:

Taverne

Please check the document version of this publication:

- A submitted manuscript is the version of the article upon submission and before peer-review. There can be important differences between the submitted version and the official published version of record.

People interested in the research are advised to contact the author for the final version of the publication, or visit the DOI to the publisher's website.

- The final author version and the galley proof are versions of the publication after peer review.

- The final published version features the final layout of the paper including the volume, issue and page numbers.

Link to publication

\footnotetext{
General rights rights.

- You may freely distribute the URL identifying the publication in the public portal. please follow below link for the End User Agreement:

www.umlib.nl/taverne-license

Take down policy

If you believe that this document breaches copyright please contact us at:

repository@maastrichtuniversity.nl

providing details and we will investigate your claim.
}

Copyright and moral rights for the publications made accessible in the public portal are retained by the authors and/or other copyright owners and it is a condition of accessing publications that users recognise and abide by the legal requirements associated with these

- Users may download and print one copy of any publication from the public portal for the purpose of private study or research.

- You may not further distribute the material or use it for any profit-making activity or commercial gain

If the publication is distributed under the terms of Article 25fa of the Dutch Copyright Act, indicated by the "Taverne" license above, 


\section{MODEL-BASED COST-EFFECTIVENESS OF CONVENTIONAL AND INNOVATIVE CHEMO-RADIATION IN LUNG CANCER}

\author{
Mathilda L. Bongers \\ Department of Epidemiology and Biostatistics, VU University Medical Center \\ Dirk de Ruysscher \\ Department of Radiation Oncology, University Hospitals Leuven/ KU Leuven \\ Cary Oberije \\ Philippe Lambin \\ Department of Radiation Oncology (MAASTRO), GROW Research Institute, Maastricht University \\ Medical Centre
}

\author{
Carin A. Uyl-de Groot \\ Institute for Medical Technology Assessment, Erasmus University Rotterdam \\ José Belderbos \\ Department of Radiotherapy, The Netherlands Cancer Institute, Antoni van Leeuwenhoek Hospital \\ Veerle M.H. Coupé \\ Department of Epidemiology and Biostatistics, VU University Medical Center \\ v.coupe@vumc.nl
}

Introduction: Optimizing radiotherapy with or without chemotherapy through advanced imaging and accelerated radiation schemes shows promising results in locally advanced non-small-cell lung cancer (NSCLC). This study compared the cost-effectiveness of positron emission tomography-computed tomography based isotoxic accelerated sequential chemo-radiation (SRT2) and concurrent chemo-radiation with daily low-dose cisplatin (CRT2) with standard sequential (SRTI) and concurrent chemo-radiation (CRT1).

Methods: We used an externally validated mathematical model to simulate the four treatment strategies. The model was built using data from $200 \mathrm{NSCLC}$ patients treated with curative sequential chemo-radiation. For concurrent strategies, data from a meta-analysis and a single study were included in the model. Costs, utilities, and resource use estimates were obtained from literature. Primary outcomes were the incremental cost-effectiveness and cost-utility ratio (ICUR) of each strategy. Scenario analyses were carried out to investigate the impact of uncertainty.

Results: Total undiscounted costs and quality-adjusted life-years (QALYs) for SRTI, CRT1, SRT2, and CRT2 were EUR 17,288, EUR 18,756, EUR 19,072, EUR 17,360 and QALYs 1.10, 1.15, 1.40, and 1.40, respectively. Compared with SRT1, the ICURs were EUR 38,024/QALY for CRTI, EUR 6,249/QALY for SRT2, and EUR 346/QALY for CRT2. CRT2 was highly cost-effective compared with SRT1. Moreover, CRT2 was more effective and less costly than CRT1 and SRT2. Therefore, these strategies were dominated by CRT2. Conclusion: Optimized sequential and concurrent chemo-radiation strategies are more effective and cost-effective than the current conventional sequential and concurrent strategies. Concurrent chemo-radiation with a daily low dose cisplatin regimen is the most cost-effective treatment option for locally advanced inoperable NSCLC patients.

Keywords: Cost-effectiveness, Modeling, Chemo-radiation, Radiotherapy, Lung cancer

Lung cancer remains a major cause of death worldwide with over 1 million deaths a year (1). Non-small-cell lung cancer (NSCLC) represents 80 percent of all lung tumors. Approximately 35 percent of the patients presents with locally advanced nonmetastatic disease. In The Netherlands, this amounts to roughly 1,000 NSCLC patients yearly presenting with inoperable stage III disease. These patients are mostly treated with (chemo-) radiotherapy with curative intent. According to the Dutch guidelines, treatment with concurrent chemotherapy and radiotherapy is preferred if patients are considered fit enough, while sequential treatment ought to be considered for frailer or older patients (2).

Overall, 55 percent of Dutch inoperable stage III patients receive concurrent chemo-radiotherapy, versus 45 percent se-

This research was performed within the framework of CTMM, the Center for Translational Molecular Medicine, project AIRFORCE (grant 030-103). Study sponsors had no role in the design of the study, the execution of the project, or the writing of the manuscript. quential treatment. There is level I evidence that concurrent chemo-radiotherapy decreases local recurrences and mortality compared with sequential chemo-radiotherapy. However, concurrent chemo-radiotherapy is substantially more burdensome for patients than sequential chemo-radiotherapy, resulting in more acute esophagitis and hematological toxicity (3). In addition, recommendations for concurrent chemo-radiotherapy are based on pooled analysis of trials with relatively fit patients with minimal co-morbidities (4). These patients are not representative of the typical NSCLC patient population. Finally, new developments in chemo-radiotherapy necessitate a renewed evaluation of the optimal treatment choice.

Because of technical developments in radiotherapy treatment planning and delivery, it became possible to deliver isotoxic dose prescriptions, whereby doses of radiotherapy are individualized according to the constraints of the organs at risk (4). In addition, PET-imaging is used to guide tumor delineation as tumor activity of the primary tumor and the affected lymph nodes are more accurately visualized compared with CT-scans. 
Pilot studies have shown the benefits of such individualized radiation schemes. Further optimization of radiotherapy, obtained by means of accelerated and hyperfractioned schemes, has been shown to improve 5-year survival in nonconcurrent schedules (5).

In addition, there is a focus on optimization of the chemotherapy schemes to minimize toxicity while maintaining the beneficial effect of a concurrent scheme on local control and overall survival. Koning et al. (2013) found that a concurrent scheme with a daily low-dose cisplatin reduced toxicity as compared to standard doublet cisplatin-based chemotherapy while maintaining activity (6).

Different sequential and concurrent chemo-radiotherapy schemes have been studied in diverse trials with different inclusion criteria. Therefore, direct comparison of the health outcomes is not possible. However, mathematical modeling gives the opportunity to bring together different treatment schemes in one consistent framework, to compare health effects, while correcting for differences in patient population. In essence, under a set of explicit assumptions, a mathematical model can be used to simulate outcomes of a randomized trial where this has not been done in actuality (7).

With the increasing costs of cancer care and limited resources available, it is important to consider the costeffectiveness of new treatments to support decision-making. In this study, we used a NSCLC micro-simulation model that we developed and described previously (8). Transitions in the micro-simulation model depend on a set of patient and tumor features, which makes it possible to simulate patient populations with a different case-mix. The micro-simulation model was used to obtain 3-year health and cost predictions under different concurrent and sequential chemo-radiation schemes.

\section{METHODS}

The aim of this study was to compare health outcomes and costs of four different sequential and concurrent chemo-radiotherapy strategies. We used an externally validated micro-simulation model, built using observational data. This model was previously used to assess the cost-effectiveness of PET-based isotoxic accelerated radiotherapy treatment, either alone or with sequential chemotherapy, compared with conventional (CTbased) fixed dose radiotherapy (9). For the current study, this model was adapted, such that the model could be used to evaluate other chemo-radiotherapy strategies.

\section{The Chemo-radiotherapy Strategies under Evaluation}

We compared the following sequential and concurrent chemoradiotherapy strategies: (i) Sequential chemo-radiotherapy (SRT1): After chemotherapy, patients received a radiation dose of $60 \mathrm{~Gy}$, in once-daily 2 Gy fractions, 5 times per week. (ii) Concurrent chemo-radiotherapy (CRT1): Patients received radiotherapy concurrently with chemotherapy. Radiotherapy

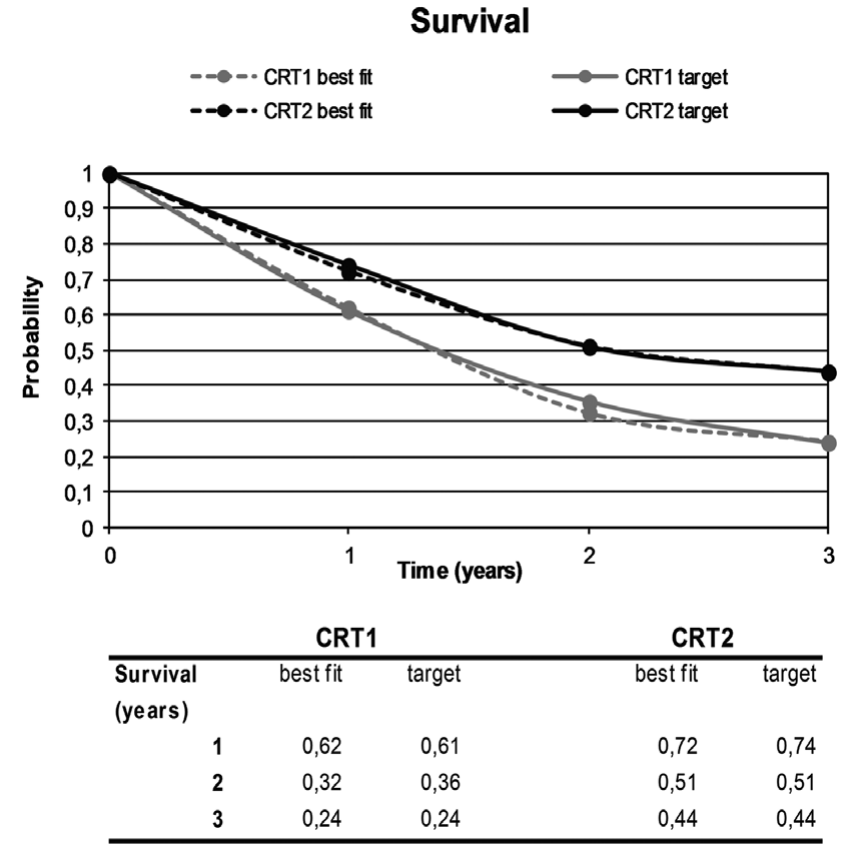

Figure 1. Model predictions of $0 S$ when using the best fitting set of parameters resulting from the calibration procedure for CRTI and CRT2 (dotted lines), as well as the calibration targets used (solid lines). Note that for the calibration procedure as well as for the internal validation, the case-mix of patients in the simulated cohort is similar to the case-mix of patients in the original data source.

started at the beginning of the second course of chemotherapy with a dose of $60 \mathrm{~Gy}$, in daily 2 Gy fractions, 5 times per week. (iii) Sequential isotoxic accelerated chemo-radiotherapy (SRT2): After chemotherapy, patients received a radiation dose of 54.0-79.2 Gy, delivered in 1.8 Gy fractions, twice daily. The term isotoxic refers to the individualization of the exact radiation dose according to the constraints of the organs (lungs and spinal cord) at risk. The mean overall treatment time was 25 days. (iv) Concurrent accelerated chemo-radiotherapy (CRT2): Patients received 66 Gy in once-daily 2.75 Gy fractions in a mean overall treatment time of 32 days. In addition, patients received cisplatin $\left(6 \mathrm{mg} / \mathrm{m}^{2}\right) 1-2$ hours before each fraction.

The first two treatment strategies (SRT1 and CRT1) are conventional strategies, in the sense that they reflect current common practice. SRT2 represents an individualized, optimized, radiotherapy scheme, and CRT2 a treatment scheme with an unusually low daily dose of chemotherapy to minimize toxicity. Patients that received isotoxic radiotherapy (SRT2) were assumed to receive an additional PET-CT scan for treatment planning in the treatment position.

\section{Model Description}

The model contains four clinical states from alive to death, with intermediate states "local recurrence" and "distant metastasis," and allows five possible transitions. Supplementary Figure 1 presents the structure of the model. All patients start in state "Alive," where they received treatment. Over time, they can develop a local recurrence "LR" (transition 1), a distant 
metastasis "DM" (transition 2), or they can die without the detection of a LR or DM "Death" (transition 3). After if they move to LR or DM, they are again at risk for Death (transition 4 and 5 , respectively).

The model simulates the disease trajectories of individual patients by repeatedly sampling a patient profile, consisting of patient and tumor characteristics (WHO performance status, gross tumor volume [GTV], N status, sequentially treated with chemotherapy) that are randomly drawn from prespecified correlated distributions. Once a specific individual patient profile is drawn, progression to subsequent health states in the model is simulated under a given treatment. The transitioning of patients between health states is governed by personalized hazard rates. These hazard rates consist of a baseline time-dependent hazard that is the same for each individual, and a personal timeindependent hazard rate ratio (HR) that varies according to the characteristics of the sampled patient profile and the treatment given. These parameters were estimated using statistical modeling (8), as described in more detail in Supplementary Table 1 and illustrated in Supplementary Figures 2 and 3. Probabilities to acquire different toxicities were included in the model (1012). Model parameters are presented in Table 1 . The time horizon was 3 years. Model predictions for overall survival were internally validated for each of the four strategies. The initial micro-simulation model was validated externally for overall survival (8). The micro-simulation model was programmed in Excel 2003 and the Visual Basic Editor 2003.

\section{Data Used for Model Quantification}

The original micro-simulation model was built using data of 200 NSCLC patients with inoperable stage I-IIIB receiving curative radiotherapy. Patients in the dataset were referred to the Maastro Clinic in Maastricht, The Netherlands, between 2002 and 2009. Data were collected prospectively (13). Patients in the dataset received either conventional radiotherapy (according to the scheme of SRT1) or PET-CT-based accelerated radiotherapy (scheme as in SRT2), with or without induction chemotherapy. Patients in the data for SRT1 and SRT2 were stage I-III patients, in contrary to the patients in the literature (CRT1 and CRT2), which were mainly stage III patients. However, the proportion of patients in the Maastro clinic that were stage I-II was 15 percent only. The distribution of patients per strategy is presented in Supplementary Table 2.

Model parameters were estimated by multi-state statistical modeling, using conventional radiotherapy as the comparator strategy. That means that five HRs, one for each of the five possible transitions in the model, specify the effect of PETCT-based accelerated radiotherapy relative to the comparator strategy. As the addition of chemotherapy was included as a covariate in the model, sequential chemo-radiotherapy strategies could be simulated with the model. Because the model was not developed for simulation of concurrent chemo-radiotherapy, the five HRs for both CRT1 and CRT2, specifying the treatment effect relative to the comparator strategy (SRT1), were obtained by calibration of model output against either the literature or study data, as described below (14).

\section{Model Calibration to Quantify Treatment Effect of the Concurrent Strategies}

Model calibration involves systematic adjustment of model parameters such that model-predicted outcomes correspond to observed outcomes. For the two treatment strategies CRT1 and CRT2, the five HRs, specifying the treatment effect relative to SRT1 for each of the five possible transitions in the model could not be estimated directly from patient-level data. Therefore, calibration was used to obtain estimates for these HRs. That is, for CRT1 and CRT2, respectively, we repetitively drew random sets of values for the $5 \mathrm{HRs}$ from within a plausible range. Subsequently, the model was used to generate predictions of 1-, 2-, and 3-year survival.

These were directly compared with observed survival outcomes by calculating the sum of squared errors of the differences in survival. The best fitting set was defined as the set of HRs that minimizes this sum of squared errors. For CRT1 and CRT2, respectively, model-predictions were compared with survival outcomes taken from a meta-analysis and from one particular study $(15 ; 16)$. The study that informed CRT2 provided information on baeline characteristics of the population investigated and observed toxicities under CRT2. In addition, the authors provided us with additional information on 1-, 2-, and 3-year survival. Observations for the proportions of local recurrence and metastasis were not available for all strategies, thus the model was calibrated to fit overall survival only. For more details on the calibration, we refer to Supplementary Table 3.

Table 1 shows the best fitting sets of HRs for CRT1 and CRT2 that were the result of the calibration procedure. Figure 1 shows the fit of the model predictions against the calibration targets for both CRT1 and CRT2. Validation of the model for the sequential treatment strategies is also described in Supplementary Table 3 and depicted in Supplementary Figure 4.

\section{Inclusion of Toxicities in the Model}

For the probability to acquire dysphagia, the micro-simulation model included a statistical ordered logit model with the covariates "N-stage" and " \pm isotoxic." Setting " \pm isotoxic" to either 0 (SRT1) or 1 (SRT2) produces risk estimates to acquire dysphagia for both treatment strategies. The risk to acquire no or a specific grade of dyspnea in the model was based on probabilities directly derived from the data for SRT1 and SRT2, respectively $(10 ; 11)$. Because no data were available to estimate the occurrence of dyspnea and dysphagia in CRT1 and CRT2, literature was used $(3 ; 16)$. For the concurrent schemes, the hematological toxicities neutropenia and anemia were added to the model (3). See Supplementary Table 1 for further details. 
Hazard ratios for each transition (point estimate, SE)

\begin{tabular}{lcccccc} 
Multi-state model & 1: Alive to LR & 2: Alive to DM & 3: Alive to death & 4: LR to death & 5: DM to death & Source \\
\hline WHO ( $\geq 2$ versus 0/1) & $2,34(0,5)$ & $2,31(0,32)$ & $1,72(0,45)$ & & \\
N stage $(\geq 2$ versus 0/1) & $0,75(0,43)$ & $1,51(0,27)$ & $2,98(0,35)$ & & \\
SRT2 & $0,47(0.41)$ & $0,10(0,37)$ & $0,40(0,33)$ & $0,70(0,44)$ & $0,87(0,23)$ & Data (13) \\
CRT1 & 0,71 & 0,92 & 0,70 & 0,66 & 0,84 & $(3)$ \\
CRT2 & 1,70 & 0,28 & 0,16 & 0,29 & 0,84 & $(16)$ \\
Chemotherapy & $2,37(0,00)$ & $3,21(0,00)$ & $0,60(0,00)$ & & \\
GTV (per 10 cc) & $1,01(0,47)$ & $1,02(0,34)$ & $1,03(0,34)$ & &
\end{tabular}

\begin{tabular}{lccccc} 
Toxicities & Dysphagia $\geq$ grade 3 & Dyspnea $\geq$ grade 3 & Neutropenia $\geq$ grade 3 & Anemia $\geq$ grade 3 & Source (year) \\
\hline SRT1 & 0,02 & 0,05 & 0,49 & 0,21 & Data (13), (3) \\
SRT2 & 0,05 & 0,10 & 0,49 & 0,21 & Data (13), (3) \\
CRT1 & 0,26 & 0,04 & 0,64 & 0,17 & $(3)$ \\
CRT2 & 0,15 & 0,07 & 0,03 & 0,00 & $(16)$ \\
Proportion of dyspnea that is irreversible & & 0,71 & & $(4)$
\end{tabular}

SRT1, conventional sequential chemo-radiation; CRI1, conventional concurrent chemo-radiation; SRT2, sequential PET-CT-based isotoxic accelerated chemo-radiation; CRT2, concurrent chemo-radiation with daily low-dose cisplatin administration and daily radiation.

SE, standard error; WHO, WHO performance status; N stage, number of lymph nodes affected; GTV, gross tumor volume; LR, local recurrence; DM, metastasis.

\section{Costs and Health-Related Quality of Life}

We performed the analysis from the health care setting of the Netherlands, using a health system perspective for the costeffectiveness analysis. That is, we included costs of radiochemotherapy, cost of treating adverse events, and all related costs, such as hospital admission, medication, and follow-up visits (17-21). Costs of chemotherapy were based on the treatment schemes presented in the literature. Per scheme we calculated the vials needed for a patient with an average body surface of $1.7 \mathrm{~m}^{2}$. For the daily dose cisplatin administration, the infusion takes approximately 1 hour daily. In Supplementary Table 4, a detailed overview of the costs of chemotherapy is provided. For SRT2, costs of an additional PET-scan were included. All costs were standardized to 2014 Euros, using the consumer price index.

To estimate quality of life, we used utility estimates derived from the literature $(17 ; 22 ; 23)$. Once a patient in the model obtained anemia, neutropenia, dysphagia, or dyspnea grade 3, or dyspnea and dysphagia grade 2 simultaneously, a disutility value of 0.353 was assigned for the duration of the adverse events. Duration of both dyspnea and dysphagia was fixed at 30 days. Severe dyspnea is assumed to be irreversible for 71 percent of the patients. For those patients, we assumed that quality of life was decreased by the disutility value for adverse events of 0.353 for the entire time horizon in the model. A full overview of resource use, costs, (dis)utilities, and the sources we used is presented in Table 2.

\section{Base-Case Analysis}

For the base-case analysis, the disease history of a hypothetical cohort of patients under all four strategies was simulated with a distribution of baseline clinical features and tumor features as observed in the database from the Maastro Clinic for nonmetastatic NSCLC patients ineligible for surgery, that received (sequential chemo-)radiotherapy with curative intent (18). Patients in the simulation had a relatively good WHO performance status ( 88 percent had a status of 0 or 1 ), 75 percent of patients had two or more lymph nodes affected and the mean GTV was $77 \mathrm{cc}^{3}$. In all strategies, the distribution of clinical and tumor features was held constant to eliminate bias due to difference in baseline characteristics.

Model predictions were obtained by simulating the health trajectories of a cohort of 50,000 patients from the moment they start treatment until they die or have reached the timehorizon of 3 years. This time horizon was chosen because it was deemed appropriate to cover the health benefits and costs of radiotherapy in NSCLC. Additionally, in the base-case analysis the following assumptions were made: (i) For each type of chemo-radiotherapy strategy, the treatment affects all 
Table 2. List of Input Parameters for Resource Use, Unit Costs, and Utilities.

\begin{tabular}{|c|c|c|c|}
\hline & Point estimate & SE (alpha, beta) & Source \\
\hline \multicolumn{4}{|l|}{ Resource use } \\
\hline \multicolumn{4}{|l|}{ Radiotherapy } \\
\hline Number of fractions SRTI/CRTI & 31 & 1,89 & Data (13) \\
\hline Number of fractions SRT2 & 36 & 5,71 & Data (13) \\
\hline Number of fractions CRT2 & 24 & 5,71 & (16) \\
\hline Contouring PET-imaging (hours) & 1,5 & & Expert opinion \\
\hline \multicolumn{4}{|l|}{ Adverse events } \\
\hline Days of medication dysphagia & 30 & & (17) \\
\hline Days of hospital admission with dysphagia & 2 & $(13,33 ; 0,15)$ & (17) \\
\hline Days of tube feeding with dysphagia & 22 & $(220,50 ; 0,10)$ & (17) \\
\hline Days of irreversible dyspnea & lifelong & & \\
\hline \multicolumn{3}{|l|}{ Outpatient Visits } & (17) \\
\hline Year 1 & 4 & & \\
\hline Year 2 & 2 & & \\
\hline Year 3 & 1 & & \\
\hline \multicolumn{3}{|l|}{ Costs (EUR) } & (Source) year of conversion \\
\hline \multicolumn{4}{|l|}{ Treatment } \\
\hline Radiotherapy per fraction & 244 & 16,95 & 2010 \\
\hline PET-scan & 1325 & & (9) 2012 \\
\hline PET contouring, per hour & 54,25 & & Based on yearly income 'laborant' \\
\hline Outpatient visits & 60 & & (18) 2010 \\
\hline \multicolumn{3}{|l|}{ Chemotherapy (total costs) } & Supplementary Table 4 \\
\hline SRTl & 2768 & & \\
\hline SRT2 & 2768 & & \\
\hline CRTl & 4014 & & \\
\hline CRT2 & 6590 & & \\
\hline End-of-life care costs in cancer per year (palliative care only) & 6603 & 737 & (19) 2000 \\
\hline \multicolumn{4}{|l|}{ Toxicity } \\
\hline Medication dysphagia per day & 2.80 & & (17) 2012 \\
\hline Treatment irreversible dyspnea per year & 1140 & 112 & 2000 \\
\hline Replacing/removing tube & 105 & & (18) 2009 \\
\hline Tube feeding per day & 25 & & (17) 2009 \\
\hline Antibiotics, per course & 83 & & (20) 2008 \\
\hline Blood transfusion & 88 & & (21) 2006 \\
\hline G-CSF, per course & 1,737 & & (20) 2008 \\
\hline Hospital admission per night & 462 & & (18) 2010 \\
\hline Outpatient day & 263 & & (18) 2010 \\
\hline \multicolumn{3}{|l|}{ Utilities } & Source \\
\hline Reference value & 0,82 & 0,067 & $(22)$ \\
\hline Disutility any adverse event RT & 0,35 & 0,05 & $(17,23)$ \\
\hline Disutility hematologic toxicity & 0.24 & & $(17,23)$ \\
\hline
\end{tabular}

SRT1, conventional sequential chemo-radiation; CRT1, conventional concurrent chemo-radiation; SRT2, sequential PET-CT-based isotoxic accelerated chemo-radiation; CRT2, concurrent chemo-radiation with daily low-dose cisplatin administration and daily radiation; SE, standard error; RT, radiotherapy; G-CSF, granulocyte colony-stimulating factor. 
Table 3. Base-Case Scenario: Model Predictions for the Four Radiotherapy Strategies over a 3-Year Time Horizon $(N=50,000)$ and ICERs and ICURs Compared to the Reference Strategy and Compared to the Next Best Cost-Effective Strategy.

\begin{tabular}{|c|c|c|c|c|}
\hline & SRTI & CRTl & SRT2 & CRT2 \\
\hline \multicolumn{5}{|l|}{ Survival (\%) } \\
\hline 1 year & 0.49 & 0.60 & 0.62 & 0.65 \\
\hline 2 years & 0.20 & 0.29 & 0.39 & 0.44 \\
\hline 3 years & 0.18 & 0.21 & 0.30 & 0.37 \\
\hline \multicolumn{5}{|c|}{ Recurrence at 3 years $(\%)$} \\
\hline LR & 0.13 & 0.08 & 0.11 & $\overline{0.28}$ \\
\hline DM & 0.62 & 0.66 & 0.50 & 0.51 \\
\hline \multicolumn{5}{|c|}{ Toxicity at 3 years (\%) } \\
\hline Dysphagia & 0.04 & 0.26 & 0.09 & 0.15 \\
\hline Dyspnea & 0.05 & 0.04 & 0.10 & 0.07 \\
\hline Neutropenia & 0.42 & 0.55 & 0.42 & 0.03 \\
\hline Anemia & 0.18 & 0.15 & 0.18 & 0 \\
\hline \multicolumn{5}{|c|}{ Undiscounted LYS, QALYS, and costs at 3 years } \\
\hline Total LY & 1.38 & 1.44 & 1.77 & 1.78 \\
\hline Total QALYs & 1.10 & 1.15 & 1.40 & 1.40 \\
\hline Total costs $(€)$ & 17288 & 18756 & 19072 & 17360 \\
\hline \multicolumn{5}{|c|}{ Discounted LYs, QALYS, and costs at 3 years } \\
\hline Total LY & 1.37 & 1.42 & 1.75 & 1.75 \\
\hline Total QALYS & 1.09 & 1.13 & 1.38 & 1.38 \\
\hline Total costs $(€)$ & 17156 & 18627 & 18958 & 17257 \\
\hline \multicolumn{5}{|c|}{ Cost-effectiveness and cost-utility compared to SRTl } \\
\hline ICER $(€ / L Y)$ & & 29814 & 4708 & 263 \\
\hline ICUR (€/QALY) & & 38024 & 6249 & 346 \\
\hline \multicolumn{5}{|c|}{ Incremental analysis: cost-effectiveness and cost-utility compared to next best strategy } \\
\hline ICER $(€ / L Y)$ & NA & Dominated $^{0}$ & Dominated $^{\mathrm{a}}$ & $263^{b}$ \\
\hline ICUR (€/QALY) & NA & Dominated $^{a}$ & Dominated $^{a}$ & $346^{b}$ \\
\hline
\end{tabular}

\footnotetext{
aThrough extended dominance.

bCompared to SRTI.

NA, not applicable; SRTI, conventional sequential chemo-radiation; CRTI, conventional concurrent chemo-radiation; SRT2, sequential PET-CT-based isotoxic accelerated chemo-radiation; CRT2, concurrent chemo-radiation with daily low-dose cisplatin administration and daily radiation; Cl, confidence interval; ICER, incremental cost effectiveness ratio; LY, life-years; ICUR, incremental cost-utility ratio; QALYS, quality-adjusted life-years; LR, local recurrence; DM, distant metastasis.
}

transitions and this effect is different for each transition. (ii) In all treatment strategies, patients have similar resource use after the end of chemo-radiotherapy treatment (with the exception of toxicity-related resource use). (iii) Toxicities can overlap. (iv) All deaths within the time horizon are caused by cancer.

Model outcomes included predictions of survival, and proportions of patients experiencing dysphagia, dyspnea, neutropenia and anemia in the treatment period. Costs(discounted), life-years (LYs) lived and quality-adjusted life-years (QALYs) lived within a 3-year time horizon are the main model outcomes used for the cost-effectiveness calculations. A time horizon of 3 years was deemed appropriate for this patient group, because in the data more than 80 percent of lung can- cer deaths had already occurred within this period. For discounting of the results, a 3 percent discount rate per year was used.

The incremental cost-effectiveness ratio (ICER) and incremental cost-utility ratio (ICUR) were calculated as the ratio between the difference in costs and the difference in either LYs or QALYs between each strategy and the comparator strategy. SRT1 was used as the comparator strategy. In addition, an incremental analysis was done, in which all strategies were compared with the next best nondominated strategy. A strategy is dominated if there is an alternative strategy that is more effective at equal or lower costs. We used the term "dominant" if a strategy was more effective at equal or lower costs than all other strategies evaluated. 
According to the World Health Organization, a strategy is considered highly cost effective if the ICUR does not exceed the value of once the gross domestic product per capita purchasing power parity (GDP) of a country. This means a threshold value of EUR 30,491 per QALY in Europe (24).

\section{Scenarios and Sensitivity Analyses}

To assess the impact of uncertainty in model parameters on the results of the cost-effectiveness analysis, ideally a probabilistic sensitivity analysis is carried out. However, when combining a statistical model with calibration, the uncertainty of the calibrated parameters as well as the correlation among all parameters in the model is unknown, precluding a mathematically sound probabilistic sensitivity analysis. To assess the possible consequences of model uncertainties, we, therefore, carried out two scenario analyses and four sensitivity analyses. First, we obtained model predictions for a different patient cohort, that is, a cohort with patients in better physical condition; we assumed that all patients had a WHO performance status of 0 or 1 , and that 75 percent of the patients had more than two affected lymph nodes. We assumed that the mean GTV was $100 \mathrm{cc}^{3}$. In general, this profile was seen in patients eligible for concurrent chemo-radiation in data and literature (13). In the second scenario, we set the costs and disutilities for toxicities to zero.

In the first sensitivity analysis, we studied the impact of using alternative sets of five HRs for the treatment effect of SRT2, CRT1, and CRT2 compared with SRT1. To be more specific, we selected for SRT2, CRT1, and CRT2 sets of HRs that resulted in model predictions corresponding to the lower and upper band of the 95 percent confidence interval (CI) of the data-based survival curve.

Furthermore, we decreased disutilities for toxicities with 50 percent (sensitivity analysis 2 ) and varied the costs of treatment for toxicities with -50 percent and +50 percent (sensitivity analysis 3 ). Because costs of end-of-life care were assigned to patients that died within the time horizon of the model, we also varied the costs of end-of-life care with -50 percent and +50 percent (sensitivity analysis 4 ).

\section{RESULIS}

\section{Base-case Scenario}

The results of the base-case scenario in which the distribution of clinical and tumor features was held constant over the four strategies, are presented in Table 3. Predicted discounted average survival in the base-scenario was 1.37 LY for SRT1, 1.42 LY for CRT1, 1.75 LY for SRT2, and 1.75 LY for CRT2. The discounted QALYs followed the same ranking, from 1.09 for SRT1, 1.13 for CRT1, 1.38 for SRT2, and 1.38 for CRT2.

Discounted costs of SRT1 were estimated to be EUR 17,156, followed by CRT2 (EUR 17,257), CRT1 (EUR 18,627), and SRT2 (EUR 18,958). Costs for radiotherapy were highest in SRT1 (EUR 8,716) and lowest in CRT2 (EUR 5,864) due to the number of fractions in each strategy. Costs of chemotherapy were highest in CRT2 (EUR 6,590) and lowest in SRT1 and SRT2 (both EUR 2,768). Other cost differences are the additional PET-scan for treatment planning in SRT2 (EUR 1,406) and costs of end-of-life care, which are highest in SRT1 (EUR 5,645) and lowest in CRT2 (EUR 4,191). A break-down of the costs in the base-case analysis for each strategy can be found in Supplementary Table 5.

As shown in Table 3, the ICER and ICUR compared with reference strategy SRT1, were EUR 29,814/LY and EUR 38,024/QALY for CRT1, EUR 4,708/LY and EUR 6,249/QALY for SRT2, and EUR 263/LY and EUR 346/QALY for CRT2, respectively. CRT1 was dominated by SRT2 and CRT2 because it produces fewer LYs gained (or QALYs) at higher costs than both these strategies. Considering the aforementioned threshold value of EUR 30,439, SRT2 and CRT2 can be considered highly cost-effective compared with SRT1. However, CRT2 was the most cost-effective strategy. This strategy was not only highly cost-effective compared with reference strategy SRT1, but also more or as effective and less costly than the other two alternative strategies.

\section{Scenarios and Sensitivity Analyses}

The impact of the scenario and sensitivity analyses on discounted costs and QALYs are summarized in the tornado diagrams in Supplementary Figure 5. Additional results concerning ICERs and ICURs compared with SRT1 and those resulting from the incremental analyses can be found in Supplementary Table 6 . When the four strategies were evaluated for a patient cohort with a different case-mix, we found that CRT2 dominated both SRT1 and CRT1. SRT2 was slightly more effective than CRT2, but the ICUR compared with CRT2 was EUR 55,746 per QALY, which is above the Dutch willingness-topay threshold of EUR 30,491 per QALY. In scenario 4, with costs and disutilities for toxicities set to zero, cost-differences between the concurrent strategies and SRT1 were affected compared with the base-case analysis, but the overall costeffectiveness results were very similar.

Of all sensitivity analyses, the first analysis in which CRT1, SRT2, and CRT2 were simulated with parameter sets reflecting the lower and upper CI for survival, had the largest impact on cost- and QALY differences. Nevertheless, in all sensitivity analyses, we found similar rankings for incremental costeffectiveness as in the base-case scenario. That is, CRT2 was either the dominant strategy (being more effective and less costly than all other strategies), or was highly cost-effective compared with SRT1.

\section{DISCUSSION}

Compared with conventional sequential chemo-radiotherapy (SRT1), sequential isotoxic accelerated chemo-radiotherapy 
as well as standard concurrent chemo-radiotherapy and concurrent chemo-radiotherapy with a daily low-dose cisplatin scheme are more effective and slightly costlier. In general, cost-differences between strategies were small. Concurrent chemo-radiotherapy with daily low-dose cisplatin was the most effective strategy, and was also less costly than standard chemo-radiotherapy and sequential isotoxic accelerated chemo-radiotherapy. This means that the latter two strategies were dominated by concurrent chemo-radiotherapy with daily low-dose cisplatin. When assuming conservative treatment effects, we found that concurrent chemo-radiotherapy with daily low-dose cisplatin is still the most effective and cost-effective strategy.

\section{Existing Evidence}

Where SRT1 and CRT1 are fully implemented strategies in the treatment of NSCLC, SRT2 aims at an individually escalated dose and CRT2 aims to enhance the effects of irradiation by the radio sensitizing effect of cisplatin. For these strategies evidence is available regarding improved local tumor control. Evidence on survival and quality of life is limited $(25 ; 26)$. This is the first study to assess the comparative cost-effectiveness of these specific chemo-radiation schemes. By using a model-based analysis, we predicted effectiveness and cost-effectiveness on clinical outcomes, such as survival and quality of life outcomes, and costs. We found two modelbased study studies that evaluated radiotherapy schemes in the literature $(27 ; 28)$. One study found that a hyperfractionated and accelerated scheme was cost-effective compared with conventional radiotherapy in Belgium (27). Another study compared various types of accelerated and hyperfractionated schemes, and found that modified schemes were more effective than conventional radiotherapy, at higher costs (28). However, none of these studies assessed a strategy with an isotoxic dosing scheme or a low-dose chemotherapy scheme specifically.

\section{Model Challenges}

The micro-simulation model that was used in this study was based on a multi-state statistical model that was directly derived from data. To adapt the model to allow simulation of the four chemo-radiotherapy schemes, we used calibration. Our approach is new because it brings together statistical modeling techniques and calibration to assess the required parameters in a micro-simulation model. This new approach came with several challenges. First, the micro-simulation model was initially quantified with data on patients that either received sequential chemo-radiotherapy or received radiotherapy alone. To verify validity of the model for patients that receive sequential chemoradiotherapy only, we obtained model predictions for this particular subgroup of patients and compared those with outcomes for the corresponding subset of patients in the data. The results of this model validation are presented in the Supplementary
Materials. Overall, model fit is reasonable, although the shape of the model-predicted survival curves differs from the databased survival curves, probably mainly due to semi-parametric assumptions underlying the micro-simulation model.

Second, the micro-simulation model did not include parameters specifying the effect of concurrent chemo-radiation compared with the reference strategy for the five model transitions. These parameters were estimated by calibration. The calibration targets were obtained from the literature. It should be noted that in the meta-analysis that was used for calibration of CRT1, two studies were included that used a daily low dose cisplatin regimen, comparable to our CRT2 strategy. Therefore, the results of CRT1 may be a bit too optimistic. The treatment effects of both CRT1 and CRT2 were calibrated while keeping the other covariate effects in the model (WHO performance status, lymph node status, GTV) constant. It is probably not fully correct to assume that parameter estimates for the other covariates in the model hold also in the presence of the concurrent treatment effects.

Third, although potential differences in baseline characteristics were taken into account in the model, not all factors causing heterogeneity were included. For example, staging of patients was slightly different over the treatment strategies. The histology of patients, as well as age and proportion of females was similar over the four strategies.

Fourth, an important advantage of using a statistical model to inform the entire micro-simulation model is that parameter uncertainty can be assessed while accounting for the joint correlation between parameters. When combining a statistical model with calibration, however, the actual uncertainty of the calibrated parameters is unknown. Therefore, a mathematically sound probabilistic sensitivity analysis taking the correlation among parameters into account is no longer possible. It was shown previously that ignoring the correlation among parameters when carrying out a probabilistic sensitivity analysis of a microsimulation model leads to uncertainty bounds that are excessively inflated (29). To assess the possible consequences of model uncertainties, we, therefore, carried out scenario and sensitivity analyses. In all these analyses, we found a similar ranking in costs and effects as in the base-case-scenario.

\section{Study Limitations}

This study has several limitations. First, the health-care perspective was used instead of a societal perspective. Therefore, loss of productivity at work was not incorporated, nor the patient burden related to the number of hospital visits for each (chemo-)radiotherapy administration. Second, we used a time horizon of 3 years instead of a lifelong time horizon. However, because in the data more than 80 percent of lung cancer deaths had already occurred within 3 years, this time horizon seems appropriate for this patient group. Finally, our study was carried out in a Dutch health-care setting, using mainly Dutch sources 
for costs and resource use. Although costs vary widely over countries due to varying health care settings, the results of the scenario analyses showed that varying cost parameters does not change the ranking in cost-effectiveness, and does not change the treatment decision. Therefore, we believe that if this study was conducted in another country with different unit costs, the ranking in cost-effectiveness of the treatment strategies is likely to be similar to our base-case scenario.

\section{Study Results in the Dutch Context}

According to the Dutch guidelines, eligible patients for chemotherapy and radiotherapy are preferentially treated with concurrent chemotherapy and radiotherapy. In less fit patients, sequential chemo-radiotherapy is given and obviously the older or frail patients are treated with radiation alone or palliatively (30).

This study provides indications that sequential isotoxic accelerated chemo-radiotherapy is a more effective and costeffective treatment option than conventional concurrent or sequential chemo-radiotherapy. However, concurrent chemoradiotherapy with a daily low-dose cisplatin regimen may be a better option for physically fit nonmetastatic NSCLC patients ineligible for surgery, because of improved survival, quality of life, and cost-effectiveness.

Data from the Dutch Cancer Registry (www.iknl.nl) indicate that, in The Netherlands, roughly 1,000 inoperable stage III NSCLC patients are yearly treated with either concurrent (55 percent) or sequential (45 percent) chemo-radiotherapy. If the conventional concurrent regimen would be replaced with concurrent chemo-radiotherapy with a daily low-dose cisplatin regimen, this would amount to yearly cost-savings of roughly EUR 770,00 while gaining 138 QALYs. If the yearly 450 NSCLC patients who currently receive conventional sequential chemo-radiotherapy would receive sequential isotoxic accelerated chemo-radiotherapy, this would cost EUR 800,00 while gaining 135 QALYs. In case the latter subgroup would tolerate the concurrent low-dose cisplatin scheme, because of decreased toxicity compared with the conventional scheme, this would cost only 32,000 Euro yearly for the same health gain of 135 QALYs.

\section{CONCLUSIONS}

Optimized sequential and concurrent chemo-radiation strategies are more effective and cost-effective than conventional sequential and concurrent strategies. Concurrent chemo-radiation with a daily low-dose cisplatin regimen is the most costeffective treatment option for nonmetastatic NSCLC patients ineligible for surgery. For patients, that are physically not fit enough to receive concurrent chemo-radiation, sequential isotoxic accelerated chemo-radiotherapy is a good and costeffective alternative option.

\section{SUPPLEMENTARY MATERIAL}

Supplementary Figure 1:

https://doi.org/10.1017/S0266462317000939

Supplementary Table 1:

https://doi.org/10.1017/S0266462317000939

Supplementary Figure 2:

https://doi.org/10.1017/S0266462317000939

Supplementary Figure 3:

https://doi.org/10.1017/S0266462317000939

Supplementary Table 2:

https://doi.org/10.1017/S0266462317000939

Supplementary Table 3:

https://doi.org/10.1017/S0266462317000939

Supplementary Figure 4:

https://doi.org/10.1017/S0266462317000939

Supplementary Table 4:

https://doi.org/10.1017/S0266462317000939

Supplementary Table 5:

https://doi.org/10.1017/S0266462317000939

Supplementary Figure 5:

https://doi.org/10.1017/S0266462317000939

Supplementary Table 6:

https://doi.org/10.1017/S0266462317000939

\section{CONFLICTS OF INTEREST}

Dr. Bongers reports grants from GlaxoSmithKline and from AIRFORCE Consortium during the conduct of the study, other from Astellas Pharma Europe outside the submitted work. Drs. De Ruysscher, Oberije, and Lambin have nothing to disclose. Dr. Uyl-de Groot reports grants from Center for Translation Molecular Medicine during the conduct of the study, grants from Boehringer-Ingelheim outside the submitted work. Dr. Belderbos has nothing to disclose. Dr. Coupé reports grants from CTMM Center for Translational an Molecular Medicine during the conduct of the study, grants from Novartix outside the submitted work.

\section{REFERENCES}

1. Parkin DM, Bray F, Ferlay J, Pisani P. Global cancer statistics, 2002. $C A$ Cancer J Clin. 2005;55:74-108.

2. Vansteenkiste J, De Ruysscher D, Eberhardt WEE, et al. Early and locally advanced non-small-cell lung cancer (NSCLC): ESMO Clinical Practice Guidelines for diagnosis, treatment and follow-up. Ann Oncol. 2013;24(Suppl 6):vi89-vi98.

3. O'Rourke N, Roqué I Figuls M, Farré Bernadó N, Macbeth F. Concurrent chemoradiotherapy in non-small cell lung cancer. Cochrane Database Syst Rev. 2010:CD002140.

4. De Ruysscher D, Faivre-Finn C, Nestle U, et al. European Organisation for Research and Treatment of Cancer recommendations for planning and delivery of high-dose, high-precision radiotherapy for lung cancer. J Clin Oncol. 2010;28:5301-5310.

5. Mauguen A, Le Pechoux C, Saunders MI, et al. Hyperfractionated or accelerated radiotherapy in lung cancer: An individual patient data metaanalysis. J Clin Oncol. 2012;30:2788-2797. 
6. Koning CC, Wouterse SJ, Daams JG, et al. Toxicity of concurrent radiochemotherapy for locally advanced non-small-cell lung cancer: A systematic review of the literature. Clin Lung Cancer. 2013;14: 481-487.

7. Drummond MF. Methods for the economic evaluation of health care programmes. Cambridge: Oxford University Press; 2005:404.

8. Bongers ML, De Ruysscher D, Oberije C, et al. Multi-state statistical modeling: A tool to build a lung cancer micro-simulation model that includes parameter uncertainty and patient heterogeneity. Med Decis Making. 2016;36:86-100.

9. Bongers ML, Coupé VHM, De Ruysscher D, et al. Individualized PETbased isotoxic accelerated radiotherapy is cost effective compared to conventional radiotherapy: A model-based evaluation. Int J Radiat Oncol Biol Phys. 2015;91:857-865.

10. Dehing-Oberije C, De Ruysscher D, Petit S, et al. Development, external validation and clinical usefulness of a practical prediction model for radiation-induced dysphagia in lung cancer patients. Radiother Oncol. 2010;97:455-461.

11. Dehing-Oberije C, De Ruysscher D, van Baardwijk A, et al. The importance of patient characteristics for the prediction of radiation-induced lung toxicity. Radiother Oncol. 2009;91:421-426.

12. De Ruysscher D, Dehing C, Yu S, et al. Dyspnea evolution after highdose radiotherapy in patients with non-small cell lung cancer. Radiother Oncol. 2009;91:353-359.

13. Dehing-Oberije C, Yu S, De Ruysscher D, et al. Development and external validation of prognostic model for 2-year survival of non-small-cell lung cancer patients treated with chemoradiotherapy. Int J Radiat Oncol Biol Phys. 2009;74:355-362.

14. Vanni T, Karnon J, Madan J, et al. Calibrating models in economic evaluation: a seven-step approach. Pharmacoeconomics. 2011;29: 35-49.

15. Aupérin A, Le Péchoux C, Rolland E, et al. Meta-analysis of concomitant versus sequential radiochemotherapy in locally advanced non-smallcell lung cancer. J Clin Oncol. 2010;28:2181-2190.

16. Uyterlinde W, Belderbos J, Baas C, et al. Prediction of acute toxicity grade $\geq 3$ in patients with locally advanced non-smallcell lung cancer receiving intensity modulated radiotherapy and concurrent low-dose Cisplatin. Clin Lung Cancer. 2013;14:541548.

17. Grutters JPC, Pijls-Johannesma M, Ruysscher DD, et al. The costeffectiveness of particle therapy in non-small cell lung cancer: Exploring decision uncertainty and areas for future research. Cancer Treat Rev. 2010;36:468-476.
18. Tan SS, Bouwmans CA, Rutten FF, Hakkaart-van Roijen L. Update of the Dutch Manual for Costing in Economic Evaluations. Int J Technol Assess Health Care. 2012;28:152-158.

19. Polder JJ, Barendregt JJ, van Oers H. Health care costs in the last year of life-The Dutch experience. Soc Sci Med. 2006;63:1720-1731.

20. Timmer-Bonte JNH, Adang EMM, Termeer E, Severens JL, TjanHeijnen VCG. Modeling the cost effectiveness of secondary febrile neutropenia prophylaxis during standard-dose chemotherapy. J Clin Oncol. 2008;26:290-296.

21. Timmer-Bonte JNH, Adang EMM, Smit HJM, et al. Cost-effectiveness of adding granulocyte colony-stimulating factor to primary prophylaxis with antibiotics in small-cell lung cancer. J Clin Oncol. 2006;24:29912997.

22. Sturza J. A review and meta-analysis of utility values for lung cancer. Med Decis Making. 2010;30:685-693.

23. Nafees B, Stafford M, Gavriel S, Bhalla S, Watkins J. Health state utilities for non small cell lung cancer. Health Qual Life Outcomes. 2008;6:84.

24. Marseille E, Larson B, Kazi DS, Kahn JG, Rosen S. Thresholds for the cost-effectiveness of interventions: alternative approaches. Bull World Health Organ. 2015;93:118-124. doi: http://dx.doi.org/10.2471/BLT.14. 138206

25. De Ruysscher D, van Baardwijk A, Steevens J, et al. Individualised isotoxic accelerated radiotherapy and chemotherapy are associated with improved long-term survival of patients with stage III NSCLC: A prospective population-based study. Radiother Oncol. 2012;102:228-233.

26. Belderbos J, Uitterhoeve L, van Zandwijk N, et al. Randomised trial of sequential versus concurrent chemo-radiotherapy in patients with inoperable non-small cell lung cancer (EORTC 08972-22973). Eur J Cancer. 2007;43:114-121.

27. Lievens Y, Kesteloot K, Van den Bogaert W. CHART in lung cancer: Economic evaluation and incentives for implementation. Radiother Oncol. 2005;75:171-178.

28. Ramaekers BLT, Joore MA, Lueza B, et al. Cost effectiveness of modified fractionation radiotherapy versus conventional radiotherapy for unresected non-small-cell lung cancer patients. J Thorac Oncol. 2013;8:1295-1307.

29. Whyte S, Walsh C, Chilcott J. Bayesian calibration of a natural history model with application to a population model for colorectal cancer. Med Decis Making. 2011;31:625.

30. Driessen EJ, Bootsma GP, Hendriks LE, et al. Stage III non-small cell lung cancer in the elderly: Patient characteristics predictive for tolerance and survival of chemoradiation in daily clinical practice. Radiother Oncol. 2016;121:26-31. 